\title{
Radioiodine Therapy of Thyroid Disease
}

\author{
WILLIAM H. BEIERWALTES \\ Division of Nuclear Medicine, Department of Internal Medicine, University of Michigan Medical Center, \\ Ann Arbor, MI 48109, U.S.A.

\begin{abstract}
Ten "ideal" steps used at the University of Michigan to treat well-differentiated thyroid cancer are presented. Using this in 103 patients with well-differentiated thyroid carcinoma and metastases outside their neck, those that were freed of their disease after ${ }^{131}{ }^{I}$ therapy survived three times as long as those not cured of their disease. Patients successfully cured of their metastases showed better conformity to the "ideal" steps than the patients with residual metastases. Each of the most commonly asked questions about ${ }^{131}$ I treatment of thyroid carcinoma following surgical treatment are discussed.
\end{abstract}

\section{Introduction}

Many patients with well-differentiated thyroid carcinoma die of their disease. It is the conviction of the author that almost no patient with well-differentiated thyroid carcinoma need die of his/her disease.

A review of the literature (Harness et al., 1974) shows that $75 \%$ of patients with all types of thyroid cancer and distant metastases die within 5 years of the diagnosis. We have followed this high risk group (103 patients with metastases outside their neck from well-differentiated thyroid carcinoma) for up to 35 years to determine the effectiveness of treatment at University of Michigan (Beierwaltes et al., 1982).

The treatment of well-differentiated thyroid cancer at our institution has been divided into a ten step protocol to assess the rate of conformity to these procedures and determine the effect of conformity on cure rate. The lack of conformity indicates the procedure was not performed because either the patient had not been asked to have the procedures, or died before the procedures were instituted.

The 10 "ideal" steps in treatment (followed since 1947)

1. Thyroidectomy within 1 year of the detection of a goiter.

2. Lobectomy and isthmasectomy with a frozen section; if carcinoma is found, an immediate contralateral lobectomy.

3. Off oral T4 and T3 post surgery for 6 weeks before imaging.

4. Do radioiodide imaging 6 weeks post surgery.

5. (a) If significant residual uptake is seen in the thyroid bed, treat with $150 \mathrm{mCi}$ of radioiodine to ablate the uptake in this "remnant".

(b) Treat with $175 \mathrm{mCi}$ of radioiodine if there is uptake in the cervical nodes (metastases). (c) Treat with $200 \mathrm{mCi}(7.4 \mathrm{GBq})$ if there are metastases outside the neck.

6. Administer replacement T4 between check-ups.

7. Re-check at 1 year post radioiodine treatment with the patient off all thyroid hormone for 6 weeks.

8 . If the 1 year scan post treatment radioiodine image is negative, re-check in 2 years.

9. If the 3 year radioiodine image is negative, re-check every 5 years thereafter with patient off all thyroid hormone for 6 weeks prior to imaging.

10. If there is recurrence of radioiodine uptake at any time we treat with $200 \mathrm{mCi}$ of iodine.

\section{Study}

One hundred and three (103) patients with welldifferentiated thyroid carcinoma and metastases outside their neck were treated using our treatment protocol. Those considered free of their metastatic disease after ${ }^{131} \mathrm{I}$ therapy survived three times longer than those not cured of their disease. Patients freed of their metastases showed better conformity with the treatment protocol than those patients not demonstrating cure of their metastases.

\section{Age of the Patient and "Cure"}

\section{Papillary carcinoma}

Those freed of their disease (average age 20) were half the age of those with residual cancer (age 45). This strongly supports the argument to aggressively treat young patients with papillary carcinoma of the thyroid while there is still a chance for "cure".

\section{Follicular carcinoma}

Those patients successfully freed from their disease (average age 36 ) were about two-thirds the age of 
those not cured of their disease (age 57). Again, this is strong evidence that younger patients with follicular carcinoma should be treated more aggressively since "cure" may be possible.

\section{Leukemia and Second Cancers}

None of the patients developed and/or died from leukemia and there was no increased incidence of second cancers. These results are in striking contrast to the reported 90 to 170 -fold increased risk of acute leukemia at 2 years post treatment of carcinoma of the overy with cancer chemotherapy (Beierwaltes, 1981; Chabner, 1977).

\section{Time from Original Surgery (Histopathological Diagnosis) to First detection of Metastases Outside of the Neck}

At the time of the original surgery $39.4 \%$ of the patients did not have thyroid cancer metastases detected. The metastases were first detected outside the neck $7.44 \mathrm{y}$ after the initial surgery with a range of 1-25 years. This is an important observation because, if inadequate surgery is performed and the patient is only followed without ablating the radioiodine uptake in the thyroid bed, it cannot be determined if the patient has metastases since the metastases will not concentrate radioiodine, with rare exceptions, as long as significant uptake remains in the thyroid bed. If one takes a "conservative" approach and waits until the patient demonstrates clinical evidence of cancer elsewhere, statistically, the death rate is markedly increased and the opportunity to eradicate the patient's disease is lost.

Thus, there is strong evidence that radioiodine should be given to ablate any uptake in the thyroid bed after adequate surgery since the patient is potentially "curable" rather than adopting a "conservative" approach of simply following the patient.

\section{Thyroidectomy}

The single most important step in the treatment of thyroid cacinoma is a lobectomy and isthmectomy followed by a lobectomy of the opposite side if carcinoma is found. Cervical nodes from both the central chamber and the lateral areas should be sampled and if carcinoma is found there should be extensive node plucking. This can be done through a collar incision. If there is concern about the morbidity of bilateral lobectomy in the treatment of proven carcinoma of the thyroid, one should be aware that the only argument raised by the experienced endocrine surgeon when considering the type of surgical thyroidectomy for Grave's disease in children, is whether they should perform a $95 \%$ thyroidectomy or a bilateral total lobectomy. Since this is the only di- lemma in the surgical treatment of childhood Grave's disease, it is unclear why a surgeon should be hesitant to perform a bilateral lobectomy or isthmectomy to treat proven carcinoma of the thyroid. A complete and true total thyroidectomy eliminates the need for radioiodine therapy and in our experience, is associated with a high permanent freedom from disease. Surgeons at our institution are convinced that a total thyroidectomy may be performed with lower morbidity (in good hands) than a subtotal lobectomy. In a sub-total lobectomy, the laryngeal nerves and parathyroid glands are not as carefully isolated before resecting the thyroid tissue.

Many surgeons find that they achieve a lower morbidity with a bilatcral total lobectomy for primary carcinoma of the thyroid than from a sub-total thyroidectomy for Grave's disease. A residual uptake in the neck of less than $5 \%$ usually allows staging. Another important reason for a double lobectomy is that microscopic cancer foci are found in the contralateral lobe in $38-87 \%$ of patients. Clinical occurrence of cancer in the opposite lobe after unilateral lobectomy is $2-40 \%$. Recurrence of cancer after subtotal thyroidectomy has been shown by Mazzaferri to be twice as common than following bilateral total lobectomy (Mazzaferri and Young, 1981). It is also possible that there could be an anaplastic focus in the thyroid that when properly removed would prevent its progression to a clinically anaplastic carcinoma of the thyroid.

The hazard of recurrences has been documented in the literature. Mazzaferri and Young (1981) reported that in a 10 year follow-up of 576 patients there were 84 recurrences. All six deaths from carcinoma occurred in this group. Nineteen percent of patients with recurrences could not have their disease eradicated. Deaths in these patients began after 30 years of age. Cervical lymph node metastases were associated with increased recurrence rates. Thus, the old statistical myth of the prognosis not being worse when cervical lymph node metastases are present is incorrect. That myth arose because young people commonly have cervical node involvement and do well for several years while old people tend not to have cervical node involvement and have a shorter survival time.

Maheshwari et al. (1981) in a report of 352 patients found 97 patients with recurrent disease. One fourth of the patients with recurrent disease failed to concentrate radioiodine. Forty-four patients died of progressive thyroid carcinoma. All of the patients who died were over 40 years of age.

A delay in treatment may allow recurrence or metastases. An image at 6 weeks after surgery allows one to institute radioiodine treatment to ablate the remnant and treat micrometastases without delay. There is evidence in the animal model that delay increases the number of lung metastases. Prompt adequate surgery and radioiodine therapy allows immediate staging. Prompt adequate surgery and 
radioiodine also insures better compliance on the part of the patients.

\section{Commonly Asked Questions Concerning Therapy of Malignant Thyroid Disease}

\section{Does lack of T4 and T3 increase tumor growth}

The only hard data on this subject evolves from experiments in animal models. Sisson and Beierwaltes (1964) showed that well-differentiated papillary carcinoma of the thyroid grew the fastest in the euthyroid rat and slowest in the rat kept hypothyroid the longest. More recently it has been shown in the sarcoma of the Ajax mouse, the Lewis fibrosarcoma in C 57BL/60 mice (Kumar et al., 1979) and in mammary tumors in mice (Vonderhaar and Greco, 1982) that these observations are correct. Thus, the only controlled experiments indicate that lack of thyroid hormone decreases the growth rate of cancer.

\section{Why not continue T4 and give TSH injections?}

Although this was done in the past, it is no longer followed in most centers. There is a higher incidence of allergic reactions to TSH, the formation of neutralizing antibodies in TSH resistance, a rapid decline of serum TSH levels (short blood half-life), and a lower tumor uptake of radioiodine (Krishnamurthy and Blahd, 1977; Hays et al., 1967; Hershman and Edwards, 1972; Gershengorn et al., 1975).

3. Why not withdraw T3 for only 2 weeks and decrease the period of fatigue and cold intolerance?

We do not do this because we have had occasional patients with metastatic carcinoma of the thyroid to lung who did not show uptake of radioactive iodine after withdrawal of T3 for as long as 3 weeks but did show an excellent uptake for both diagnosis and treatment in the lungs when they had been off $\mathrm{T} 3$ for a period of 6 wceks.

\section{Why not use radioactive ${ }^{201} \mathrm{Tl}$ instead of ${ }^{131} I$ to avoid} taking the patient off thyroid hormone?

It has been demonstrated that some patients will have their metastases detected with ${ }^{201} \mathrm{Tl}$ that did not concentrate radioiodine. It has also been demonstrated that some patients will have their metastases detected with ${ }^{201} \mathrm{Tl}$ as well while they are on thyroid hormone (Harada et al., 1980). Following these original observations, abundant evidence has appeared from our laboratory and others (Varma et al., 1987) that the use of thallium is less reliable than ${ }^{131}$ I since it may also image normal lymph nodes.

\section{Why not use serum thyroglobulin (HTG) instead of the radioiodine uptake?}

There is abundant evidence that the serum HTG is increased in many thyroid diseases (Echenique et al., 1982). It is released from both benign and malignant tissue, particularly with an increasing serum TSH. It also detects non-functioning metastases. The serum
HTG is higher with bone and lung metastases than with lymph node metastases.

Schneider (1981) showed that 14 of 15 patients with thyroid cancer had serum HTG in the normal range (less than $10 \mathrm{mg} / \mathrm{mL}$ ) while on T3. High HTG levels did not distinguish residual tissue from metastases and HTG levels were greater than normal in $18 \%$ of patients with negative images. There were positive images in 14 out of 32 patients ( $44 \%$ ) where the HTG was less than $2 \mathrm{mg} / \mathrm{mL}$. The conclusions of this study were substantiated by Echenique et al. (1982) and indicates that the combination of radioiodine images and HTG were better than using either alone.

6. Why not give $30 \mathrm{mCi}(1.11 \mathrm{GBq})$ of radioiodine ablating dose for thyroid remnant rather than $150 \mathrm{mCi}$ $(5.55 \mathrm{GBq})$ ?

The original advocates of the use of $29.9 \mathrm{mCi}$ $(1.1 \mathrm{GBq})$ of ${ }^{131} \mathrm{I}$ to ablate uptake in "normal thyroid gland remnants" after surgical thyroidectomy, for the treatment of well-differentiated thyroid cancer, believed that this dose would ablate the uptake in a normal remnant (McCowen et al., 1976). Thirty-nine percent of their patients whose remnant was not ablated with $29.9 \mathrm{mCi}(1.1 \mathrm{GBq})$ and required an additional $100 \mathrm{mCi}(3.7 \mathrm{GBq})$ dose at a later time to accomplish this, demonstrated thyroid cancer in a previously judged "normal" remnant.

Two subsequent studies in which patients were followed for longer periods to check on the "ablative" effects of a $29 \mathrm{mCi}(1.1 \mathrm{GBq})$ dose of ${ }^{131} \mathrm{I}$ in normal remnants found that a second dose was required in 12 of 13 patients and 16 of 17 patients respectively (Kuni and Klingensmith, 1980; Siddiqui et al., 1981).

Four hundred and sixty-two patients with welldifferentiated thyroid cancer and ${ }^{131}$ I uptake in thyroid remnants have been studied at the University of Michigan. A $99 \%$ follow-up was achieved for a period of $15 \mathrm{y}(0.2-37.4 \mathrm{y})$ after the first ${ }^{131} \mathrm{I}$ treatment (Beierwaltes et al., 1984). Of the 267 patients with radioiodine uptake confined to the thyroid bed, 233 $(87 \%)$ had ablation from the first dose of ${ }^{131} \mathrm{I}$ using doses ranging from 100 to $200 \mathrm{mCi}(3.7-7.4 \mathrm{GBq})$. The higher the percent uptake the more difficult it was to achieve ablation. The average ablation success reported elsewhere from $30 \mathrm{mCi}(1.11 \mathrm{GBq})$ or less is approximately $60 \%$ (Sisson and Beierwaltes, 1983).

The $100-144 \mathrm{mCi}(3.7-5.3 \mathrm{GBq})$ ablative dose may furnish "adjuvant" therapy for occult metastases. There is also increasing evidence that a suboptimal radiation dose decreases the biologic half-life of subsequent radiation doses and therefore decreases the chance of curing the patient (Rawson et al., 1951; Henk et al., 1972; Benua et al., 1962; Rawson and Leeper, 1971).

\section{Why not treat with $x$-ray therapy instead of ${ }^{131} I$ ?}

We do not use external beam $x$-ray therapy before radioiodine because it decreases the $T_{1 / 2 b}$ of ${ }^{131} \mathrm{I}$ in the thyroid cancer thus diminishing the effective radi- 
ation dose from ${ }^{131}$ I (Benua et al., 1962; Rawson and Leeper, 1971). It is not effective in treating welldifferentiated thyroid cancer (Cady, 1979). It does not ablate the uptake of ${ }^{131} \mathrm{I}$ (Carr et al., 1958) and it increases the incidence of bone marrow depression after ${ }^{131}$ I treatment (Haynie and Beierwaltes, 1963). Mazzaferri and Young (1981) showed in their study of 576 patients that it adversely effects the outcome. Many investigators believe that external beam therapy should be used only in the treatment of anaplastic carcinoma of the thyroid, adenocarcinomas refractory to ${ }^{131} I$ therapy, lymphoma, and possibly in Hurthle cell and medullary thyroid carcinoma.

8. Why treat young healthy asymptomatic persons with lung metastases and at what dose of radioiodine does pulmonary fibrosis occur?

Rall et al. (1957) showed that less than $100 \mathrm{mCi}$ (3.7 GBq) of radioiodine uptake in the lungs will not induce subsequent pulmonary fibrosis. Pulmonary fibrosis has only resulted when more than $100 \mathrm{mCi}$ $(3.7 \mathrm{GBq})$ of lung uptake occurred in patients with diffuse bilateral pulmonary metastases. The highest percent uptake observed in the lungs at the University of Michigan was $35 \%$. Since we never exceed a dose of $200 \mathrm{mCi}(7.4 \mathrm{GBq})$ it has not been possible to achieve a $100 \mathrm{mCi}(3.7 \mathrm{GBq})$ treatment dose in the lungs and therefore we have never produced pulmonary fibrosis or deteriorating lung function.

9. Will ${ }^{151}$ I therapy decrease fertility or increase the incidence of birth abnormalities?

Sarkar et al. (1976) followed 40 children treated for thyroid cancer, receiving a mean total dose of $196 \mathrm{mCi}(7.25 \mathrm{GBq})$ and a maximum dose of $691 \mathrm{mCi}$ $(25.6 \mathrm{GBq})$, for a period of from 1 to $25 \mathrm{y}$. No decreased fertility or abnormal birth history has occurred.

\section{Can radioiodine cause anaplastic transformation?}

Mazzaferri and Young (1981) showed that only half of the reported patients with papillary and follicular thyroid carcinoma who developed anaplastic cancers had a documented history of previous irradiation. Solid data on anaplastic transformation has not been established.

\section{What is the recommended radioiodine dose to treat} malignant thyroid disease and its complications?

"Standard" doses. A standard adult dose of $150 \mathrm{mCi}(5.55 \mathrm{GBq})$ of ${ }^{131} \mathrm{I}$ for a thyroidal remnant is used at the University of Michigan because it has proved successful in $87 \%$ of patients who have received adequate surgery, as discussed above. We administer $175 \mathrm{mCi}(6.5 \mathrm{GBq})$ when there is demonstrated uptake of ${ }^{131} I$ in lymph nodes after extensive surgical lymph node plucking. We administer a standard adult dose of $200 \mathrm{mCi}$ (7.4 GBq) for metastases outside the neck. Several reasons support this use of a "standard" dose of $200 \mathrm{mCi}(7.4 \mathrm{GBq})$ for adults with metastases outside the neck:

(a) NRC restriction on maximum single total dose. In 1956, the Atomic Energy Commission Subcommittee on Human Use, began to include in the ${ }^{131} \mathrm{I}$ license a limit of $200 \mathrm{mCi}(7.4 \mathrm{GBq})$ on single doses for cancer therapy (Benua et al., 1962).

(b) The NRC Sub-committee on Human Use supported this limit since a study they financed failed to establish data that proved that single doses greater than $200 \mathrm{mCi}(7.4 \mathrm{GBq})$ were more effective than a $200 \mathrm{mCi}(7.4 \mathrm{GBq})$ dose. They also found that doses greater than $200 \mathrm{mCi}(7.4 \mathrm{GBq})$ were associated with an increased incidence of complications (Benua et al., 1962).

(c) Patients who have died from their thyroid carcinoma have received larger total doses than patients successfully freed of their metastases (Leeper, 1973; Phillips, 1954).

(d) It has been shown that blood radioiodine doses larger than $200 \mathrm{cGy}$ (rad) carry an increased risk of complications (primary bone marrow depression) and, it is reasonable to do studies that allow a calculation of the dose required to deliver a blood dose of $200 \mathrm{cGy}$.

The reasons we do not administer more than $200 \mathrm{mCi}$ $(74 \mathrm{GBq})$ are that:

(i) it requires a minimum of 4 days of patient study after administering the tracer dose (Leeper, 1973);

(ii) the treatment dose frequently exhibits different kinetics than the tracer dose (Benua et al., 1962);

(iii) the proponents of this procedure have demonstrated that treatment doses in excess of $200 \mathrm{mCi}$ (7.4 GBq) carry an increased risk of complications (Benua et al., 1962).

In summary, we urge more adequate surgery and ${ }^{131}$ I ablative therapy in patients under 40 years of age and with well-differentiated thyroid cancer while curable, and cessation of ${ }^{131} \mathrm{I}$ therapy when the patient develops rapidly progressive, less well-differentiated and/or hopelessly advanced disease. It is our feeling that this approach is more conservative than inadequate surgery or inadequate ${ }^{131} I$ therapy when the disease is curable; and reserve aggressive therapy only when the disease is incurable.

Acknowledgements - This work would not have been possible without the coverage of thyroid cancer patients by most of my senior and junior staff in our Nuclear Medicine Division (with special credits to J. Sisson) since 1947. Ms Vi Rhodes prepared the manuscript for publication.

\section{References}

Beierwaltes W. H. (1981) New horizons for therapeutic Nuclear Medicine in 1981. J. Nucl. Med. 22, 549.

Beierwaltes W. H., Nishiyama R. H., Thompson N. W., Copp J. E. and Kubo A. (1982) Survival time and "cure" in papillary and follicular thyroid carcinoma with distant metastases: Statistics following University of Michigan therapy. J. Nucl. Med. 23, 561 . 
Beierwaltes W. H., Rabbani R., Dmuchowski C., Lloyd R. V., Eyre P. and Mallette S. (1984) An analysis of "ablation of thyroid remnants" with I-131 in 511 patients from 1947-1984: Experience at University of Michigan. J. Nucl. Med. 25, 1287.

Benua R. S., Cicale N. R., Sonenberg M. and Rawson R. W. (1962) The relation of radioiodine dosimetry to results and complications in the treatment of metastatic thyroid cancer. Am. J. Roentgen. 87, 171.

Cady B. (1979) Risk factor analysis in differentiated thyroid cancer. Cancer 43, 810 .

Carr E. A. Jr, Dingledine W. S. and Beierwaltes W. H. (1958) Premature resort to $\mathrm{x}$-ray therapy. A common error in treatment of carcinoma of the thyroid gland. Lancet 78, 478.

Chabner B. A. (1977) Second neoplasm-A complication of cancer chemotherapy. N. Engl. J. Med. 297, 213.

Echenique R. L., Kasi L., Haynie T. P., Glenn H. J, Samaan N. A. and Hill C. S. (1982) Critical evaluation of serum thyroglobulin levels and I-131 scans in posttherapy patients with differentiated thyroid carcinoma: Concise Communication. J. Nucl. Med. 23, 235.

Gershengorn M. C., Weintraub B. D. and Robbins J. (1975) Response to oral thyrotropin releasing hormone in athyreotic patients with thyroid cancer. In Proc. 7th Int. Thyroid Conf., Boston 1975 (Eds Robbins J. and Braverman L. E.) In Excerptia Medica International Congress Series No. 378 p. 575.

Hainan K. E. (1975) The non-surgical treatment of thyroid cancer. Br. J. Surg. 62, 769

Harada T., Ito Y., Shimaoka K., Taniguchi T., Matsudo A. and Senoo T. (1980) Clinical evaluation of 201-thallium chloride scan for thyroid nodule. Eur. J. Nucl. Med. 5, 125.

Harness J. K., Thompson N. W., Sisson J. C. and Beierwaltes W. H. (1974) Differentiated thyroid carcinomas: Treatment of distant metastases. Arch. Surg. 108, 410.

Haynie T. P. and Beierwaltes W. H. (1963) Hematologic changes observed following I-131 therapy for thyroid carcinoma. J. Nucl. Med. 4, 85.

Hays M. T., Solomon D. H. and Beall G. N. (1967) Suppression of human thyroid function by antibodies to bovine thyrotropin. J. Clin. Endocrinol. Metab. 27, 540.

Henk J. M., Kirkman S. and Owen C. M. (1972) Whole body scanning and ${ }^{131} I$ therapy in the management of thyroid carcinoma. Br. J. Radiol. 45, 369.

Hershman J. M. and Edwards C. L. (1972) Serum thyrotropin (TSH) levels after thyroid ablation compared with TSH levels after exogenous bovine TSH: Implications for ${ }^{131}$ I treatment of thyroid carcinoma. J. Clin. Endocrinol. Metab. 34, 814.

Kumar M. S., Chiang T, and Deodhar S. D. (1979) Enhancing effect of thyroxine on tumor growth and metastases in syngeneic mouse tumor systems. Cancer Res. 39, 3515 .

Kuni C. C. and Klingensmith W. C. (1980) Failure of low doses of ${ }^{131}$ I to ablate residual thyroid tissue following surgery for thyroid cancer. Radiology 137, 773 .

Krishnamurthy G. T. and Blahd W. H. (1977) Human reactions to bovine TSH. J. Nucl. Med. 18, 629 (Abstr.).

Leeper R. D. (1973) The effect of ${ }^{131}$ I therapy on survival of patients with metastatic papillary of follicular thyroid carcinoma. J. Clin. Endocrinol. Metab. 36, 1143.

Maheshwari Y. K., Hill C. S. Jr, Haynie T. P., Hickey R. C. and Samaan N. A. (1981) ${ }^{131}$ I therapy in differentiated thyroid carcinoma: M.D. Anderson Hospital experience. Cancer 47,664 .

Mazzaferri E. L. and Young R. L. (1981) Papillary thyroid carcinoma: $A 10$ year follow-up report of the impact of therapy in 576 patients. Am. J. Med. 79, 511.

McCowen K. D., Adler R. A., Ghaed N., Verdon T. and Hofeldt F. D. (1976) Low dose radioiodine thyroid ablation in post surgical patients with thyroid cancer. $\mathrm{Am}$. J. Med. 61, 52 .

Pacini F., Pinchera A., Giani C., Grasso L., Doveri F. and Baschieri (1980) Serum thyroglobulin in thyroid carcinoma and other thyroid disorders: J. Endocrinol. Invest. $3,283$.

Phillips A. F. (1954) The gamma ray dose in carcinoma of the thyroid treated by radioiodine. Acta Radiol. 41, 533 .

Rall J. E., Alpers J. B., LeWallen C. G., Sonenberg M., Berman M. and Rawson R. W. (1957) Radiation pneumonitis and fibrosis: A complication of radioiodine treatment of pulmonary metastases from cancer of the thyroid J. Clin. Endocrinol. 17, 1263.

Rawson R. W., Rall J. E, and Peacock W. (1951) Limitations and indications in treatment of cancer of thyroid with radioactive iodine. J. Clin. Endocrinol. 11, 1128.

Rawson R. W. and Leeper R. D. (1971) Treatment of thyroid cancer with radioactive iodine. In Nuclear Medicine (Ed. Blahd W. H.), 2nd edn, Chap. 27, pp. 735-750.

Sarkar S. D., Beierwaltes W. H., Gill S. P. and Cowley B. I. (1976) Subsequent fertility and birth histories of children and adolescents treated with 131-I for thyroid cancer. J. Nucl. Med. 17, 460.

Schneider A. B., Line B. R., Goldman J, M, and Robbins J. (1981) Sequential serum thyroglobulin determinations, ${ }^{131}$ I scans, and ${ }^{131}$ I uptakes after triiodothyronine withdrawal in patients with thyroid cancer. J. Clin. Endocrinol. Metab. 53, 1199.

Siddiqui A. R., Edmanson J., Wellman H. H., Hamaker R. C., Lingeman R. E., Park H.-M. and Johnston C. C. (1981) Feasibility of low doses of ${ }^{131}$ I for thyroid ablation in postsurgical patients with thyroid carcinoma. Clin. Nucl. Med. 6, 158.

Singer P. A., Nicoloff J. T., Stein R. B. and Joravillo J (1978) Transient TRH deficiency after prolonged thyroid hormone therapy. J. Clin. Endocrinol. Metab. 47, 512.

Sisson J. C. and Beierwaltes W. H. (1964) The effect of thyroidectomy with and without thyroxine replacement on transplantable thyroid tumors in rats. Endocrinology 74, 925

Sisson J. C. (1983) Applying the radioactive eraser: I-13I to ablate normal thyroid tissue in patients from whom thyroid cancer has been resected. J. Nucl. Med. 24, 743.

Varma V. et al (1987) Comparative study of T1-201 and I-131 scintigraphy in postoperative metastatic thyroid cancer. In press.

Vonderhaar B. K. and Greco A. E. (1982) Effect of thyroid status on development of spontaneous mammary tumors in primiparous $\mathrm{C} 3 \mathrm{H}$ mice. Cancer Res. 42, 4553. 